\title{
Effect of International Capital Inflows on Economic Growth of Nigeria
}

\author{
Okoro, C. O. ${ }^{\# 1}$, Nzotta, S. N. ${ }^{* 2}$, Alajekwu, U. B. ${ }^{\# 3}$ \\ "Department of Banking \& Finance, Chukwuemeka Odumegwu Ojukwu University, Igbariam Campus, \\ Anambra State, Nigeria \\ *Faculty of Management Science Technology, Federal University of Technology, Owerri, Imo State, Nigeria.
}

\begin{abstract}
The study examined the effect of international capital inflows on economic growth of Nigeria for the periods, 1986 to 2016. The study employed four core channels of international capital inflows which includes foreign direct investment (FDI), official development assistance (ODA), personal remittances (REM), and external debt stock (EXTDS) into Nigeria as the explanatory variables and GDP growth rate as the dependent variable. The model of the study was hinged on the Harrod-Domar growth model and employed Johansen cointegration and Ordinary Least Square (OLS) techniques for data analyses. The result showed that international capital inflows have long run effect on economic growth of Nigeria. Specifically, the OLS revealed that FDI and REM had significant positive effects on economic growth. However, EXTDS and ODA had no significant effects on economic growth. The study further showed that international capital is a powerful tool for boosting economic growth of Nigeria $(R$-square $=71 \%)$. The recommendations among others include that policy makers should forthwith discourage the use of external debt and official development assistance in Nigeria.
\end{abstract}

Keywords: International Capital inflows, Economic Growth, Foreign Direct Investment, Remittances, HarrodDomar, Nigeria

\section{INTRODUCTION}

Investment is one important factor for ensuring economic growth and welfare of the citizens. One local means to ensure investment is domestic savings which is in its lowest in most developing countries including Nigeria. The Harrod-Domar Model has posited that the savings as well as investment rates must be between $18 \%$ - $20 \%$ to sustain a $6 \%$ growth of GDP [1]. However, like Nigeria, most less developed countries are entrapped by the vicious circle of poverty. They lack the capital resources and the incomes of the people are very low. Investment level has remained low as a result of low savings ratio via low income. At the same time, due to low incomes, the taxable capacity remains low, that is, government earnings also remain low. In such situations, the less developed countries face savings - investment gap as well as deficit in their balance of payments. These savings-investment gap so experience in most African countries, including Nigeria, can be filled by attracting foraging capital inflows via foreign direct and portfolio investment, foreign aids, and external debt, etc.

This savings - investment gap in Nigeria can be supplemented through foreign savings. The channels for attracting foreign savings are to encourage capital flows. International capital flows is the movement of capital from countries of surplus funds to countries of 'need'. A needy country is one which has limited resources for investment that is required for economic development. The attraction of international capital inflows depend on a number of features of the host economy, which include among others; its market size, level of education, institutional environment, tax laws, and overall macroeconomic and political environment [2]. Countries that measure up to these factors could attract higher international capital inflows than others. Nigeria is the largest recipient of remittances in Sub-Saharan Africa [3]. Likewise, Nigeria is one of the largestreceivers of all forms of international capital including official development assistance, foreign private investment (foreign direct and portfolio investments), remittances as well as external debts. Be this as it may, Nigeria like most developing economies have been bedevilled by the twin economic crises of mounting debt burden and foreign investment inadequacies accompanied with more than proportionate foreign direct investment income remittance[4].

Furthermore, [5] maintains that Nigeria faces immense challenges in accelerating growth, reducing poverty and meeting the Millennium Development Goals (MDGs). Unfortunately as [6] put it, the growth experience of many of the economies such as Nigeria has not been very satisfactory and as a result, they accumulate huge external debt in relation to gross domestic product and face serious debt servicing problems in terms of foreign exchange flows and thus wallop in abject poverty. It has also been argued byreference [7] that remittances provide substantial sources of foreign exchange earnings, which could be used to finance imports, and are therefore useful in stabilizing balance of payments in some countries. Reference [4] observed that 
foreign investors come in with small amount of money, which is further magnified by the depreciating exchange rates, and end up carting away huge sums of money out of the host countries in form of investment income. In addition, these funds are reinvested in the capital markets of developed countries to the detriment of domestic markets and the resulting capital flight further worsens the crisis relating to the generation of foreign investment income. Following from this background, it becomes worrisome whether all the channels of international capital inflows into Nigeria have assisted it positively.

The main objective of the study is to examine the effect of international capital inflows on economic growth of Nigeria. Specifically, the sub-objectives include:

1. Investigating the effect of foreign direct investment on economic growth of Nigeria.

2. Finding out the effect of official development assistance on economic growth of Nigeria.

3. Examining the effect of remittances on economic growth of Nigeria.

4. Evaluating the effect of external debt stock on economic growth of Nigeria.

\section{REVIEW OF RELATED LITERATURE}

The channels of international capital flows across the world include the Foreign Direct Investment (FDI), Official Development Assistance (ODA), Remittances (REM) and External debt stock (EXTDS). These channels jointly form the sources of international capital inflows into Nigeria. The combination of these funds make up the total capital investment attracted from abroad. Conceptually, investment is defined to involve funds committed to future gains (productivity and development). Thus, foreign investment can be linked to economic growth. The conceptual framework arising from the above definitions and analogy is shown in figure 1 below.

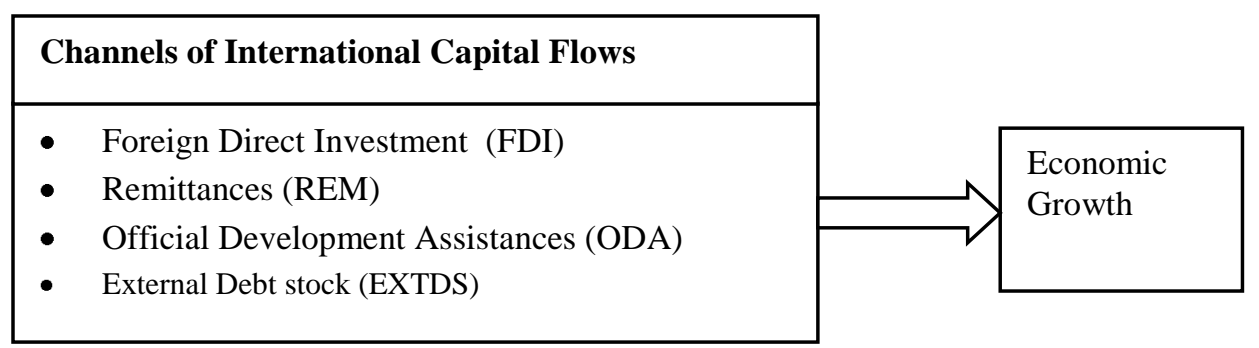

Figure 1: Channels of International Capital Inflows

\section{A. International Capital Inflow}

Several studies have used various terms to mean international capital inflow: external capital [8], foreign capital [3], [9],[10]and international capital [11]. All these terms describecapital flows from abroad into thelocal economy for productive purposes. Capital inflow is the influx of external resources into the local of capital resources for the purposes of investment, trade and business production [12]. On the other hand, investment is the funds committed into economic activity with the hope of realizing benefits from future returns over a period of time [13]. By this assertion, reference [13] connotes that investment is necessary for growth. The attraction of capital from abroad boosts local capital which is often insufficient in developing economies like Nigeria. Thus, the link between capital and growth can be gained by developing countries when they engage in encouraging and sourcing capital abroad. Sources of these capitals can be from foreign direct investment which in this study includes foreign portfolio investment, official development assistance, remittances and even external debt stock. The link between these variables and economic growth is reinvestigated because external capital inflow is necessary and sufficient for economic growth in the less developed countries [14]. Thus this study seeks to assess the possible link of the various channels of international capital inflows into Nigeria.

The major sources of international capital inflows into Nigeria are foreign direct investment, official development assistance (foreign aids), remittances, external debt as well as external trade revenue. This study aims to investigate the effect of the first four channels of capital inflows on economic growth of Nigeria. These four variables of international capital inflows are explained hereunder:

\section{B.Foreign Direct Investment (FDI)}

The terms FDI and FPI involve the process of bringing in investors resources (money, material and man) from foreign countries into the local economy for productive purposes. A combination of FDI and FPI forms foreign 
private capital flows called foreign private investment.FDI is a foreign investment made so as to acquire a lasting management interest (for instance, $10 \%$ of voting stocks) and at least $10 \%$ of equity shares in an enterprise operating in another country other than that of investors' country [15]. FDI is the direct transfer of technological know-how and managerial practices to the host country, unlike the Foreign Portfolio Investment (FPI) which is a mere change of ownership. FPI are further split between debt and equity investments and recently "financial derivatives" has been added as part of portfolio investments. In addition, available data suggest that FDI flows tendto be more stable compared to Foreign Portfolio Investment [16]. The reasons for this is that FPI has high liquidity and short time horizon because it can be traded in the financial market unlike the FDI that is in real assets. As well, the FDI inflows is hardly affected by the national exchange rate as the FPI.

\section{C.Official Development Assistance (ODA)}

Official Development Assistance (ODA) could also be called Foreign aid. ODA consists of grants or loans that one government or multilateral organization gives to a developing country to promote economic development and social welfare [17]. However, reference [18] conceptualised foreign aid (ODA) as an international transfer of capital, goods, or services for the benefit of other nations. Reference [18] further noted that these aids come in forms of capital transfers in cash or kind, either as grants or loans. Technical assistance and training usually come as grants in the form of human resources and technical equipment, and Military assistance in the form of either equipment or training advisors.

\section{D.Remittances}

The next channel of International Capital inflow is Remittances whichis money sent home by migrants working abroad to their home countries [19]. Reference [20] argues that remittances include both financial and nonfinancial materials that migrants receive while working overseas and sent back to their households in their countries of origin. In a broader sense, North-South Centre of the Council of Europe reported in reference[21] explained that remittances are ideas, practices, mind-sets, world views, values and attitudes, norms of behaviour, as well as social capital such as knowledge, experience and expertise which the citizens abroad possess and either deliberately or otherwise transfer from their host countries to their homeland.

\section{E. External Debt Stock}

The last channel of international capital inflow is the external debt stock (EXTDS) which is what the World Bank refers to as all unpaid portion of external financial resources which are needed for development purposes and balance of payment support which could not be repaid as and when due [22]. Generally, it is that portion of a country's debt that was borrowed from foreign lenders including commercial banks, governments or international financial institutions [23]; [24].

\section{F.Economic Growth}

Economic growth can be defined as the increase of per capita gross domestic product (GDP) or other measures of aggregate income typically reported as the annual rate of change in real GDP [1]. Economic growth has generally being measured in terms of Gross Domestic Product (GDP) and Human Development Index (HDI) which is an index that measures national growth based on measures of life expectancy at birth, education attainment, literacy and adjusted real per capita income [1]. The present study adopted GDP as proxy for economic growth.

\subsection{Theoretical Framework}

The theoretical framework of the study is hinged on the Harrod-Domar growth model. This is the earliest model for determining the foreign capital-growth nexus which was based on the pioneering works of the postKeynesian growth models for closed economies as designed by [25] and [26]. In their respective work, they tried to identify the pre-conditions needed to enable an industrialized economy (using a study of the U.S.) to reach steady-state equilibrium of growth. The assumption of the Harrod-Domar growth model is that labour is in excess supply and growth can only be constrained by availability and productivity of capital. The constraint to growth is caused by three gaps, namely, savings gap; trade balance gap (foreign exchange); and fiscal gap. These gaps can be filled using a foreign capital inflows to augment domestic investment.

The relationship between capital flows and saving-investment gap is captured by Keynesian macroeconomic model of an open economy which posit that national output (Y) is a function of consumption, investment, 
government spending and net export. Thus the model is: GDP $(\mathrm{Y})=$ Consumption $(\mathrm{C})+$ Investment $(\mathrm{I})+$ Government $(\mathrm{G})$ and Net Exports $(\mathrm{X}-\mathrm{M})$. This can form an equation thus: $\mathrm{Y}=\mathrm{C}+\mathrm{I}+\mathrm{G}+(\mathrm{X}-\mathrm{M})$

Also,

$\operatorname{GDP}(\mathrm{Y})=\mathrm{C}+\mathrm{S}+\mathrm{T}$

Where:

$\mathrm{C}=$ Consumption

$\mathrm{S}=$ Savings

$\mathrm{T}=$ Tax

FCR $=$ Foreign Capital Requirement

From (a) and (b)

$\mathrm{C}+\mathrm{I}+\mathrm{G}+(\mathrm{X}-\mathrm{M})=\mathrm{C}+\mathrm{S}+\mathrm{T}$

$(\mathrm{X}-\mathrm{M})=\mathrm{C}+\mathrm{S}+\mathrm{T}-\mathrm{C}-\mathrm{I}-\mathrm{G}$

$(\mathrm{X}-\mathrm{M})=\mathrm{S}-\mathrm{I}+\mathrm{T}-\mathrm{G}$

$(\mathrm{X}-\mathrm{M})=(\mathrm{S}+\mathrm{T}-\mathrm{G})-\mathrm{I}$

$\mathrm{FCR}=(\mathrm{X}-\mathrm{M})=(\mathrm{S}+\mathrm{T}-\mathrm{G})-\mathrm{I}$

In equation (f), the gap between aggregate domestic savings (private and public) and domestic investment is equal to the gap between exports and imports. The Two-gap model postulates that if the foreign exchange gap $(\mathrm{X}-\mathrm{M})$ required for achieving a target rate of growth is greater than the domestic savings-investment gap, foreign aid is needed to fill the foreign exchange gap. Similarly, foreign aid is needed to fill the savingsinvestment gap if it is the larger of the two gaps. The foreign capital requirement (FCR) in the economy could be articulated in terms of the gap between aggregate domestic saving (private and public) and domestic investment and the gap between exports and imports-eqn. (f). This theory posits that capital inflows affect economic growth positively. This theory supports the present study because the inflow of capital from the four channels of FDI, ODA, REM and EXTDS are expected to spur growth.

\subsection{Empirical Studies}

TABLE 1: REVIEW OF EMPIRICAL STUDIES

\begin{tabular}{|c|c|c|c|c|c|c|}
\hline $\begin{array}{l}\mathbf{S} / \\
\mathbf{N}\end{array}$ & $\begin{array}{l}\text { Author } \\
\text { \& Year }\end{array}$ & Country & Topic & Variables & $\begin{array}{l}\text { Method of } \\
\text { Analysis }\end{array}$ & Findings \\
\hline 1 & {$[12]$} & $\begin{array}{l}\text { Nigeria, } \\
\text { Ghana and } \\
\text { India (1986- } \\
\text { 2012) }\end{array}$ & $\begin{array}{l}\text { Impact of } \\
\text { Capital } \\
\text { Inflows on } \\
\text { Economic } \\
\text { Growth of } \\
\text { Developing } \\
\text { Countries }\end{array}$ & $\begin{array}{l}\text { RGDP=f(FDPI,FA,WRT, } \\
\text { FB) } \\
\text { Where: } \\
\text { RGDP = Real Gross } \\
\text { Domestic Product; } \\
\text { FDPI=Foreign Direct and } \\
\text { Portfolio Investment; } \\
\text { FA= Foreign Aids; } \\
\text { WRT= Workers' } \\
\text { Remittances; } \\
\text { FB= Foreign Borrowings }\end{array}$ & $\begin{array}{l}\text { Johansen } \\
\text { Co- } \\
\text { Integration, } \\
\text { Granger } \\
\text { Causality } \\
\text { and OLS } \\
\text { tests }\end{array}$ & $\begin{array}{l}\text { Capital Inflows have } \\
\text { positive influence on } \\
\text { economic growth of } \\
\text { Nigeria, Ghana and India. } \\
\text { In Nigeria and Ghana, } \\
\text { FDPI and FB have } \\
\text { significant and positive } \\
\text { impact on economic } \\
\text { growth. WRT has } \\
\text { significant positive } \\
\text { influence economic } \\
\text { growth of all the } \\
\text { countries. }\end{array}$ \\
\hline 2 & [27] & $\begin{array}{l}33 \text { Sub- } \\
\text { Sahara } \\
\text { African } \\
\text { countries } \\
(1970 \text { to } \\
2010)\end{array}$ & $\begin{array}{l}\text { Capital } \\
\text { Inflows and } \\
\text { Outflows and } \\
\text { Economic } \\
\text { Growth in } \\
\text { Sub-Sahara } \\
\text { Africa }\end{array}$ & $\begin{array}{l}\text { GDP=f(REMIT, FDI, ODA, } \\
\text { DEBT, CF, TFL, } \\
\text { SCHENROL, POP, GFCF) } \\
\text { REMIT= Ratio of } \\
\text { remittance to GDP; } \\
\text { FDI= Ratio of net FDI } \\
\text { inflows to GDP; } \\
\text { ODA= Proportion of total } \\
\text { ODA received to GDP; } \\
\text { TFL=Total Capital } \\
\text { Inflow(computed by }\end{array}$ & Sys-GMM & $\begin{array}{l}\text { FDI and Remittances } \\
\text { contributed significantly } \\
\text { to growth with } \\
\text { remittances taking the } \\
\text { lead. However, Capital } \\
\text { Flight and Debt } \\
\text { constituted significant } \\
\text { drag on growth with the } \\
\text { exact effect of ODA being } \\
\text { uncertain. }\end{array}$ \\
\hline
\end{tabular}




\begin{tabular}{|c|c|c|c|c|c|c|}
\hline & & & & $\begin{array}{l}\text { subtracting CF from the } \\
\text { summation of FDI, ODA, } \\
\text { remittances and debt); } \\
\mathrm{CF}=\text { Ratio of CF to GDP; } \\
\text { DEBT= change in the total } \\
\text { debt outstanding as a share } \\
\text { of GDP; } \\
\text { POP= population growth } \\
\text { rate; } \\
\text { GFCF=ratio of Gross Fixed } \\
\text { Capital Formation to GDP; } \\
\text { SCHENROL=School } \\
\text { enrolment, Secondary as a } \\
\text { percentage of GDP }\end{array}$ & & \\
\hline 3 & {$[28]$} & $\begin{array}{l}\text { Nigeria } \\
\text { (1981 to } \\
2012)\end{array}$ & $\begin{array}{l}\text { External } \\
\text { Capital Flows } \\
\text { and } \\
\text { Economic } \\
\text { Growth In } \\
\text { Nigeria }\end{array}$ & $\begin{array}{l}\text { RGDP=f(EXDT, FDI, STC) } \\
\text { where: } \\
\text { RGDP= Real Gross } \\
\text { Domestic Product; EXDT= } \\
\text { External Debt; FDI=Foreign } \\
\text { Direct Investment ; STC= } \\
\text { Short Term Capital inflows }\end{array}$ & $\begin{array}{l}\text { Vector Error } \\
\text { Correction } \\
\text { Mechanism( } \\
\text { VECM)tech } \\
\text { nique }\end{array}$ & $\begin{array}{l}\text { Categorization of Foreign } \\
\text { Capital inflows into direct } \\
\text { and portfolio has } \\
\text { significant effect on } \\
\text { economic growth in } \\
\text { Nigeria. External debt has } \\
\text { the strongest impact on } \\
\text { economic growth in } \\
\text { Nigeria among the foreign } \\
\text { capital factors }\end{array}$ \\
\hline 4 & [29] & $\begin{array}{l}\text { Ethopia } \\
(1960 \mathrm{~m}- \\
2010)\end{array}$ & $\begin{array}{l}\text { Impact of } \\
\text { Capital } \\
\text { Inflows on } \\
\text { Economic } \\
\text { Growth and } \\
\text { Self } \\
\text { Employment } \\
\text { in Ethiopia }\end{array}$ & RGDP=f(FDI, DAC) & $\begin{array}{l}\text { Descriptive } \\
\text { Statistics } \\
\text { and Granger } \\
\text { Causality } \\
\text { Wald Tests }\end{array}$ & $\begin{array}{l}\text { FDI has direct positive } \\
\text { effect on Real GDP in the } \\
\text { short run, but no evidence } \\
\text { that it has a direct positive } \\
\text { effect on self-employment } \\
\text { while Development } \\
\text { assistance (DAC) has no } \\
\text { direct impact on real GDP } \\
\text { but has a positive effect } \\
\text { on self-employment. }\end{array}$ \\
\hline 5 & [30] & $\begin{array}{l}\text { Bangladesh } \\
\text { (2003 to } \\
2014)\end{array}$ & $\begin{array}{l}\text { Impact of } \\
\text { Remittances } \\
\text { and FDI on } \\
\text { Economic } \\
\text { Growth: A } \\
\text { Panel Data } \\
\text { Analysis }\end{array}$ & $\begin{array}{l}\text { RGDPPC=f(GCF, } \\
\text { ENROLL, GOVTCONS, } \\
\text { CREDIT, REMIT, FDI) } \\
\text { where: } \\
\text { RGDPPC=Real GDP per } \\
\text { Capita } \\
\text { GCF= Gross Capital } \\
\text { Formation as proxy for } \\
\text { physical capital investment; } \\
\text { ENROL= Secondary Sch } \\
\text { enrolment as proxy for } \\
\text { Human capital; } \\
\text { GOVTCONS=Govt final } \\
\text { consumption expenditure as } \\
\text { a proxy for fiscal policy; } \\
\text { Credit=financial sector } \\
\text { development; } \\
\text { Remit=Remittances and } \\
\text { FDI=net foreign direct } \\
\text { investment }\end{array}$ & $\begin{array}{l}\text { Statistic and } \\
\text { Dynamic } \\
\text { Panel Data } \\
\text { Approach, } \\
\text { Pooled } \\
\text { Ordinary } \\
\text { Least } \\
\text { Square } \\
\text { method, } \\
\text { Fixed and } \\
\text { Random } \\
\text { Effect } \\
\text { Model and } \\
\text { Generalized } \\
\text { Moments } \\
\text { Methods(G } \\
\text { MM) }\end{array}$ & $\begin{array}{l}\text { FDI has significant } \\
\text { positive impact in } \\
\text { economic growth of the } \\
33 \text { developing countries } \\
\text { studied. } \\
\text { Remittance has significant } \\
\text { negative effect on the } \\
\text { economic growth across } \\
\text { the developing countries. }\end{array}$ \\
\hline 6 & [8] & $\begin{array}{l}\text { Kenya } \\
(1970 \text { to } \\
2013)\end{array}$ & $\begin{array}{l}\text { Effects of } \\
\text { External } \\
\text { Capital } \\
\text { Inflows on } \\
\text { Economic } \\
\text { Growth in } \\
\text { Kenya }\end{array}$ & $\begin{array}{l}\text { Real GDP= f(FDI, ODA, } \\
\text { REM) }\end{array}$ & $\begin{array}{l}\text { Ordinary } \\
\text { Least } \\
\text { Square } \\
\text { method and } \\
\text { Dickey- } \\
\text { Fuller } \\
\text { Augmented } \\
\text { test( to test } \\
\text { the }\end{array}$ & $\begin{array}{l}\text { Findings revealed that: } \\
\text { FDI and GDP do not have } \\
\text { a significant relationship; } \\
\text { ODA has a positive } \\
\text { significant relationship } \\
\text { with GDP; while Migrant } \\
\text { remittances and GDP has } \\
\text { a positive significant } \\
\text { relationship }\end{array}$ \\
\hline
\end{tabular}




\begin{tabular}{|c|c|c|c|c|c|c|}
\hline & & & & & $\begin{array}{l}\text { stationarity } \\
\text { of the time } \\
\text { series data }\end{array}$ & \\
\hline 7 & [10] & $\begin{array}{l}\text { Pakistan } \\
(1972-2013)\end{array}$ & $\begin{array}{l}\text { Foreign } \\
\text { Capital } \\
\text { Inflows and } \\
\text { Economic } \\
\text { Growth in } \\
\text { Pakistan: An } \\
\text { Empirical } \\
\text { Analysis }\end{array}$ & $\begin{array}{l}\mathrm{G}=\mathrm{f}(\mathrm{D}, \mathrm{F}, \mathrm{R}, \mathrm{I}, \mathrm{P}, \mathrm{L}) \text { Where: } \\
\mathrm{G}=\mathrm{GDP} \text { Growth rate; } \mathrm{D}= \\
\text { External Debt Servicing; } \mathrm{F}= \\
\mathrm{FDI} ; \mathrm{R}=\text { Workers' } \\
\text { Remittances; } \mathrm{I}=\text { Domestic } \\
\text { Investment; } \mathrm{P}=\mathrm{GDP} \text { deflator } \\
\text { as a proxy for inflation rate; } \\
\mathrm{L}=\text { Literacy rate. All except } \\
\text { Literacy rate are taken } \\
\text { as \%age of GDP. }\end{array}$ & $\begin{array}{l}\text { Johansen } \\
\text { co- } \\
\text { integration } \\
\text { technique } \\
\text { and Granger } \\
\text { Causality } \\
\text { Test }\end{array}$ & $\begin{array}{l}\text { Foreign capital inflows } \\
\text { have negative impact on } \\
\text { economic growth in the } \\
\text { long run. Short run } \\
\text { analysis confirmed } \\
\text { unidirectional causality } \\
\text { running from debt service, } \\
\text { FDI, Bidirectional } \\
\text { causality between } \\
\text { remittances and growth } \\
\text { was found. }\end{array}$ \\
\hline 8 & [31] & $\begin{array}{l}\text { Nigeria } \\
(1983- \\
2012)\end{array}$ & $\begin{array}{l}\text { effect of } \\
\text { Capital Flows } \\
\text { on economic } \\
\text { Growth }\end{array}$ & $\begin{array}{l}\text { RGDP = F(FDIN, EXCR, } \\
\text { TRAP) } \\
\text { RGDP = economic growth, } \\
\text { FDIN = Foreign Direct } \\
\text { Investment Net flow, } \\
\text { TRAP = trade openness, } \\
\text { EXCR = exchange rate }\end{array}$ & $\begin{array}{l}\text { Augmented } \\
\text { Dickey } \\
\text { Fuller, } \\
\text { conintegrati } \\
\text { on and } \\
\text { recursive } \\
\text { residuals } \\
\text { (Cusum). }\end{array}$ & $\begin{array}{l}\text { Capital inflow has both } \\
\text { long and short run effect } \\
\text { on growth. }\end{array}$ \\
\hline 9 & [32] & $\begin{array}{l}\text { Nigeria } \\
(987-2012)\end{array}$ & $\begin{array}{l}\text { effect of } \\
\text { foreigninvest } \\
\text { ments inflows } \\
\text { on economic } \\
\text { growth }\end{array}$ & $\begin{array}{l}\text { GDP }=(\text { FDI, FPI }) \\
\text { GDP = Gross Domestic } \\
\text { Product. } \\
\text { FDI = Foreign Direct } \\
\text { Investment } \\
\text { FPI = Foreign Portfolio } \\
\text { Investment }\end{array}$ & $\begin{array}{l}\text { OLS and the } \\
\text { Granger } \\
\text { causality }\end{array}$ & $\begin{array}{l}\text { Both FDI and FPI have } \\
\text { positive and significant } \\
\text { effect on economic } \\
\text { growth }\end{array}$ \\
\hline 10 & [33] & $\begin{array}{l}\text { Nigeria } \\
(1981- \\
2012)\end{array}$ & $\begin{array}{l}\text { impact of } \\
\text { capital flows } \\
\text { on the level } \\
\text { of } \\
\text { economicgro } \\
\text { wth }\end{array}$ & $\begin{array}{l}\text { RGDP = } f \text { (SAL, SLD, } \\
\text { DPF, DPI) (1) } \\
\text { RGDP = Real Gross } \\
\text { Domestic Product } \\
\text { SAL = total stock of } \\
\text { external assets and liabilities } \\
\text { to GDP. } \\
\text { SLD = stock of liabilities as } \\
\text { a share of GDP. } \\
\text { DPF = ratio of inflows and } \\
\text { outflows of (FDI and } \\
\text { portfolio flows) to GDP. } \\
\text { DPI = ratio of inflows of } \\
\text { capital (FDI and portfolio } \\
\text { inflows) to GDP. }\end{array}$ & $\begin{array}{l}\text { Johansen } \\
\text { cointegratio } \\
\mathrm{n} \text { test, ECM } \\
\text { and variance } \\
\text { decompositi } \\
\text { on tests }\end{array}$ & $\begin{array}{l}\text { net capital flow has } \\
\text { significant positive } \\
\text { influence on the level of } \\
\text { economic growth }\end{array}$ \\
\hline 11 & [3] & $\begin{array}{l}\text { Nigeria } \\
(1982- \\
2012)\end{array}$ & $\begin{array}{l}\text { Extent to } \\
\text { which foreign } \\
\text { capital flows } \\
\text { have } \\
\text { impacted on } \\
\text { growth } \\
\text { performance }\end{array}$ & $\begin{array}{l}\text { GDP = f(Inflow + outflow } \\
\text { +Opn + Hcd + Ifr) } \\
\text { GDP = Gross Domestic } \\
\text { Product; } \\
\text { Infow=Foreign Capital } \\
\text { Inflows; } \\
\text { Outflow =Foreign Capital } \\
\text { Outflow; } \\
\text { Opn =Openness of the } \\
\text { Economy; } \\
\text { HCD=Human Capital } \\
\text { Development Index; } \\
\text { Infr. =Inflation rate; }\end{array}$ & $\begin{array}{l}\text { multiple } \\
\text { regression } \\
\text { analysis } \\
\text { method }\end{array}$ & $\begin{array}{l}\text { Foreign capital flows } \\
\text { have positive effect on } \\
\text { growth. }\end{array}$ \\
\hline 12 & [9] & $\begin{array}{l}\text { Nigeria } \\
(1981-2010)\end{array}$ & $\begin{array}{l}\text { foreign } \\
\text { capital } \\
\text { inflows } \\
\text { components } \\
\text { and economic } \\
\text { growth }\end{array}$ & $\begin{array}{l}\text { RGDPt }=\mathrm{f}(\mathrm{AID}, \mathrm{RMT}, \mathrm{FDI}, \\
\mathrm{ED}) \\
\text { where } R G D P=\text { real } G D P) \text {, } \\
\text { and } A I D, R M C \text {, FDI, and } \\
T E D \text {, represents foreign aid, } \\
\text { remittance, foreign direct }\end{array}$ & $\begin{array}{l}\text { cointegratio } \\
\mathrm{n}, \text { variance } \\
\text { decompositi } \\
\text { on and } \\
\text { impulse } \\
\text { response }\end{array}$ & $\begin{array}{l}\text { There is } \begin{array}{r}\text { significant } \\
\text { negative, }\end{array} \\
\text { positive, and negative } \\
\text { positive and } \\
\text { effect of foreign aid, } \\
\text { remittance, FDI and } \\
\text { external debt on real GDP }\end{array}$ \\
\hline
\end{tabular}




\begin{tabular}{|c|c|c|c|c|c|c|}
\hline & & & & $\begin{array}{l}\text { investment, and external } \\
\text { debt respectively. }\end{array}$ & $\begin{array}{l}\text { analysis and } \\
\text { block } \\
\text { exogeneity } \\
\text { tests }\end{array}$ & respectively \\
\hline 13 & [34] & $\begin{array}{l}\text { SSA } \\
\text { countries of } \\
\text { Nigeria, } \\
\text { Ghana and } \\
\text { South } \\
\text { Africa }\end{array}$ & $\begin{array}{l}\text { impact of } \\
\text { foreign } \\
\text { capital } \\
\text { inflows on the } \\
\text { economic } \\
\text { growth }\end{array}$ & $\begin{array}{l}\text { RGDP = f(FDI, FPI, ODA, } \\
\text { EMR, OPN, LBF, EXCHr, } \\
\text { INFL) } \\
\text { Where: } \\
\text { FDI = Foreign direct } \\
\text { investment; FPI = Foreign } \\
\text { portfolio investment; ODA } \\
\text { = Overseas development } \\
\text { assistance; EMR = } \\
\text { Economic migrant's } \\
\text { remittances; OPN = } \\
\text { Openness to foreign trade; } \\
\text { LBF = Labor Force; EXCHr } \\
\text { = Exchange Rate; INFL = } \\
\text { Inflation }\end{array}$ & $\begin{array}{l}\text { Cointegratio } \\
\mathrm{n} \text {, and ECM }\end{array}$ & $\begin{array}{l}\text { No long run effects but } \\
\text { there is short run effects }\end{array}$ \\
\hline 14 & [35] & $\begin{array}{l}\text { West Africa } \\
\text { Monetary } \\
\text { Zone } \\
\text { economies } \\
(1981-2010)\end{array}$ & $\begin{array}{l}\text { examine the } \\
\text { implications } \\
\text { of foreign } \\
\text { capital } \\
\text { inflows on } \\
\text { output growth }\end{array}$ & $\begin{array}{l}\text { RGDP = f(FDI, FPI, ODA, } \\
\text { REM, PDS, REXR, RINT) } \\
\text { RGDP is real Gross } \\
\text { Domestic Product, foreign } \\
\text { direct investment (FDI), } \\
\text { foreign portfolio investment } \\
\text { (FPI), official development } \\
\text { assistance (ODA) and } \\
\text { workers' remittances } \\
\text { (REM). And control } \\
\text { variables of PDS is private } \\
\text { domestic savings, REXR is } \\
\text { real exchange rate, RINT is } \\
\text { real interest rate }\end{array}$ & $\begin{array}{l}\text { Seemingly } \\
\text { Unrelated } \\
\text { Regression } \\
\text { Estimation } \\
\text { (SURE) } \\
\text { technique }\end{array}$ & $\begin{array}{l}\text { Foreign capital inflows } \\
\text { have a varying effect on } \\
\text { growth of } \text { WAMZ } \\
\text { countries }\end{array}$ \\
\hline 15 & [36] & $\begin{array}{l}\text { Nigeria } \\
(1981-2014)\end{array}$ & $\begin{array}{l}\text { relationship } \\
\text { between } \\
\text { foreign } \\
\text { capital } \\
\text { inflows and } \\
\text { economic } \\
\text { growth }\end{array}$ & $\begin{array}{l}\text { GDP =f(FDI, FPI, FA) } \\
\text { GDP = Gross Domestic } \\
\text { Product, } \\
\text { FDI = Foreign Direct } \\
\text { Investment, } \\
\text { FPI = Foreign Private } \\
\text { Investment, } \\
\text { FA = Foreign Aid } \\
\end{array}$ & $\begin{array}{l}\text { Toda } \\
\text { Yamamoto } \\
\text { test of } \\
\text { causality }\end{array}$ & $\begin{array}{l}\text { Increase in foreign capital } \\
\text { inflow causes GDP to } \\
\text { increase positively }\end{array}$ \\
\hline 16 & [11] & $\begin{array}{l}\text { India } \\
(1995- \\
2004)\end{array}$ & $\begin{array}{l}\text { impact of } \\
\text { international } \\
\text { capital flows } \\
\text { on financial } \\
\text { markets and } \\
\text { economic } \\
\text { growth }\end{array}$ & $\begin{array}{l}\text { IIP = f(FDI, FPI, FII) } \\
\text { Where, } \\
\text { IIP = Index of Industrial } \\
\text { Production; FDI = Foreign } \\
\text { Direct Investment; FPI = } \\
\text { Foreign Portfolio } \\
\text { Investment; FII = Foreign } \\
\text { Institutional Investment }\end{array}$ & $\begin{array}{l}\text { OLS } \\
\text { technique }\end{array}$ & $\begin{array}{l}\text { FDI is positively affecting } \\
\text { the economic growth, } \\
\text { while FII is negatively } \\
\text { affecting the growth }\end{array}$ \\
\hline
\end{tabular}

\section{Source: Authors conception}

The empirical review showed that there is a conflict with regards to the nature and direction of the effect of international capital inflow channels (FDI, FPI, ODA, REM and EXTDS) on economic growth. In some studies there were even mixed findings. For instance, the works conducted in Nigeria, Ghana and India [12]; Nigeria [28],[32],[33],[36]. However, reference [34] who studied SSA countries of Nigeria, Ghana and South Africa posited that FDI, FPI and ODA had short run effect but no long run relationship. The major conflict and gap in empirical study arises from the notion that there could be different effect from each of the different channels of international capital inflows [35]. 
A study covering 33 SSAcountries posited that Foreign Direct Investment, Foreign Portfolio Investment, and external debt have positive effect on growth but the effect of ODA is uncertain [27]. A study from Ethopia supported this by positing that all international capital inflow channels had positive effect on growth but for ODA [29]. In another study, reference [30] posited that remittances had negative effect on growth. In Kenya, [8], noted that FDI had no effect while ODA and remittances had positive effects. Even in Nigeria where most of the studies tend to support positive effects from all international capital inflow channels, [9] came up to posit that ODA and external debts had negative effects. This underscores the level of inconsistencies in the empirical findings across developing and emerging economies and Nigeria in particular. The major gap that this study intends to fill is to reexamine the international capital inflows-growth nexus in Nigeria.

\section{METHODOLOGY}

The study adopted the ex-post facto research design. The ex-post-facto design was used because the data used for this study have already been documented by highly research based institutions- the CBN. Thus, researchers have to adapt to and rely on such official publications for valid and reliable academic exercise. The source of data for this study was from World Development Indicator, 2016. The time frame covered a period of 1986 to 2016.

The study is anchored on the Harrod-Domar growth model to design a model depicting growth as a direct function of international capital inflows. However, the model of the present study equally adapted variables from the following studies:

Refinance [12]: RGDP=f(FDPI,FA,WRT, FB)

Where: RGDP = Real Gross Domestic Product; FDPI=Foreign Direct and Portfolio Investment; FA= Foreign Aids; WRT = Workers' Remittances; FB= Foreign Borrowings.

Reference [8]: Real GDP=f(FDI, ODA, REM)

Where: Real GDP = Real Gross Domestic Product; FDI = Foreign Direct Investment; ODA = Official Development Assistance and REM = Remittances.

All these previous models adopted GDP as the dependent variables and the independent variables are core international inflow variables. The models did not consider control variables. Following these models, the present study formed a complete model of international capital inflow variables that included all the four variables identified from the two studies above. The present study controlled for investment risk because of some implied economic restrictions to trade and attendant effects of liberalized and free flow of economic resources. Thus, the current model included exchange rate as follows:

GDPr $=f(F D I$, ODA, REM, EXTDS, EXR)

The function above can be rewritten into equation form as follows:

$\mathrm{GDPr}=\beta_{0}+\beta_{1} \mathrm{FDI}+\beta_{2} \mathrm{ODA}+\beta_{3} \mathrm{REM}+\beta_{4} \mathrm{EXTDS}+\beta_{5} \mathrm{EXR}+\mu$

Where:

GDPr $=$ Growth rate of real Gross Domestic Product used as a proxy for economic growth and the dependent variable in the model.

FDI = Foreign Direct Investment inflows divided by GDP used as a proxy for Foreign Direct Investment.

ODA = Official development assistance divided by GDP used as a proxy for Foreign Aid. 
$\mathrm{REM}=$ Personal remittances divided by GDP used as a proxy for remittance.

EXTDS = Total external debt stock divided by GDP used as a proxy for external debt stock .

$\mathrm{EXR}=$ Real effect exchange rate of US dollar to Nigerian Naira. $\beta_{0}$ is the constant while $\beta_{1-5}$ are the coefficients of the explanatory variables. The theoretical expectation of the study is that capital inflows have positive effect on economic growth. The relationship is $\beta_{1}>\beta_{2}>\beta_{3}>\beta_{4}>0<\beta_{5}$. The econometric tools of data analyses were employed to estimate the model posed to examine the effect of international capital inflows on economic growth of Nigeria. The tests includestationarity tests, Johansson co-integration test and OLS regression technique.

\section{PRESENTATION AND ANALYSIS OF DATA}

\section{TABLE 2: THE UNIT ROOT TEST RESULTS FOR THE SELECTED VARIABLES IN NIGERIA}

\begin{tabular}{|c|c|c|c|c|c|c|}
\hline \multirow{2}{*}{\multicolumn{2}{|c|}{ Variables }} & \multicolumn{2}{|c|}{ Levels } & \multicolumn{2}{|c|}{ First Difference } & \multirow[t]{2}{*}{ Decision } \\
\hline & & ADF & $\mathbf{P P}$ & ADF & PP & \\
\hline \multicolumn{2}{|l|}{ GDPr } & $-4.4129 *$ & $-4.3876 *$ & - & - & $1(0)$ \\
\hline \multicolumn{2}{|l|}{ FDI } & $-3.5327 *$ & $-3.5327 *$ & - & - & $1(0)$ \\
\hline \multicolumn{2}{|l|}{ ODA } & $-3.7898 *$ & $-2.8675^{* * *}$ & - & - & $1(0)$ \\
\hline \multicolumn{2}{|l|}{ REM } & -2.1013 & -2.1111 & $-5.6996^{*}$ & $-5.7448 *$ & $1(1)$ \\
\hline \multicolumn{2}{|l|}{ EXTDS } & -1.2634 & -1.4010 & $-4.1807 *$ & $-5.5510 *$ & 1(1) \\
\hline \multicolumn{2}{|l|}{ EXR } & $-3.8498 *$ & $-3.9573 *$ & - & - & $1(0)$ \\
\hline \multirow{3}{*}{$\begin{array}{l}\text { Critical } \\
\text { Values }\end{array}$} & $1 \%$ & -3.6793 & -3.6793 & -3.6998 & -3.6892 & \\
\hline & $5 \%$ & -2.9678 & -2.9678 & -2.9763 & -2.9719 & \\
\hline & $10 \% \%$ & -2.6229 & -2.6229 & -2.6274 & -2.6251 & \\
\hline
\end{tabular}

The results on Table 1 indicate that variables on GDPr, FDI, and ODA do not have unit root at level. This means that these variables are integrated in the order of I(0). This implies that GDPr, FDI, and ODAare stationary at levels. However, REM and EXTDS have unit root at 1(0); implying they are not stationary at their levels. However, the tests showed that the first difference of the variables has no unit root and the null hypothesis was rejected at $5 \%$ level of significance, indicating that all the variables are integrated of the order of first difference, that is $\mathrm{I}(1)$.

\section{Table 3: THE RESULTS OF THE LONG RUN RELATIONSHIP BETWEEN INTERNATIONAL CAPITAL INFLOW AND ECONOMIC GROWTH}

Unrestricted Cointegration Rank Test (Trace)

\begin{tabular}{ccccc}
\hline \hline $\begin{array}{c}\text { Hypothesized } \\
\text { No. of CE(s) }\end{array}$ & Eigenvalue & $\begin{array}{c}\text { Trace } \\
\text { Statistic }\end{array}$ & $\begin{array}{c}0.05 \\
\text { Critical Value }\end{array}$ & Prob.** \\
\hline \hline None * & 0.969376 & 197.4894 & 95.75366 & 0.0000 \\
At most 1* & 0.829056 & 99.88263 & 69.81889 & 0.0000 \\
At most 2* & 0.563383 & 50.42295 & 47.85613 & 0.0281 \\
At most 3 & 0.400744 & 27.21939 & 29.79707 & 0.0964 \\
At most 4 & 0.315042 & 12.88153 & 15.49471 & 0.1192 \\
At most 5 & 0.078412 & 2.286400 & 3.841466 & 0.1305 \\
\hline \hline
\end{tabular}


Unrestricted Cointegration Rank Test (Maximum Eigenvalue)

\begin{tabular}{ccccc}
\hline \hline $\begin{array}{c}\text { Hypothesized } \\
\text { No. of CE(s) }\end{array}$ & Eigenvalue & $\begin{array}{c}\text { Max-Eigen } \\
\text { Statistic }\end{array}$ & $\begin{array}{c}0.05 \\
\text { Critical Value }\end{array}$ & Prob.** \\
\hline \hline None * & 0.969376 & 97.60679 & 40.07757 & 0.0000 \\
At most 1* & 0.829056 & 49.45967 & 33.87687 & 0.0003 \\
At most 2 & 0.563383 & 23.20357 & 27.58434 & 0.1650 \\
At most 3 & 0.400744 & 14.33786 & 21.13162 & 0.3379 \\
At most 4 & 0.315042 & 10.59513 & 14.26460 & 0.1757 \\
At most 5 & 0.078412 & 2.286400 & 3.841466 & 0.1305 \\
\hline \hline
\end{tabular}

The result of the Johansson co-integration test was based on Trace statistics and Maximum Eigenvalue. Trace statistics indicate three co-integration equations while the Maximum Eigenvalue statistics showed only two cointegrating equations. The results support that the variables are co-integrated. This means that there is a long run relationship between international capital inflows and economic growth in Nigeria.

\section{Table 4: OLS REGRESSION OF THE EFFECT OF INTERNATIONAL CAPITAL INFLOWS AND ECONOMIC GROWTH}

\begin{tabular}{lrrrr}
$\begin{array}{l}\text { Dependent Variable: GDPR } \\
\text { Method: Least Squares }\end{array}$ & & & & \\
Sample: 1986 2016 & & & & \\
Included observations: 31 & & & & \\
\hline \hline Variable & Coefficient & Std. Error & t-Statistic & Prob. \\
\hline \hline FDI & 0.667553 & 0.824890 & 10.809263 & 0.0260 \\
ODA & 0.164745 & 1.010577 & 0.163021 & 0.8718 \\
REM & 0.518247 & 0.672745 & 3.770347 & 0.0183 \\
EXTDS & -0.068816 & 0.039042 & -1.762604 & 0.0902 \\
EXR & -0.027885 & 0.022862 & 11.219695 & 0.0340 \\
C & 12.88203 & 4.316274 & 2.984524 & 0.0063 \\
\hline \hline R-squared & 0.712561 & & & \\
Adjusted R-squared & 0.655073 & & & \\
F-statistic & 19.349696 & & & \\
Prob(F-statistic) & 0.006525 & & & \\
Durbin-Watson stat & 1.971283 & & & \\
\hline \hline
\end{tabular}

The result of the OLS regression is used to determine the sub-objectives of the study. It tested the hypotheses of the study. The coefficient of determination $(\mathrm{R} 2)=0.712561$ showed that about $71 \%$ of changes in economic growth in Nigeria is accounted for by the level of international capital inflows. This implies that international capital inflow is one major contributor to Nigeria's economic growth. The F-statistics $(19.349696 ;$ p. $<0.05)$ indicated that all the variables of the model (international capital inflow variables) have significant effect on economic growth. The Durbin Watson statistics (1.971283) showed that there was no autocorrelation in the model employed.

To address the specific objectives, the coefficient of regression, $t$-statistic and its corresponding p.values were used. This was aimed at finding out the effect of individual independent variables of International Capital inflows on economic growth. 


\section{Test of Hypotheses and Discussion of Findings}

The results showed FDI had a positive $(0.667553)$ and significant $(\mathrm{p}<0.05)$ effect on economic growth (GPDR). Thus the hypothesis "Foreign direct investment has no significant effect on economic growth of Nigeria" is rejected. The study, therefore, concludes that foreign direct investment has significant effect on economic growth of Nigeria. Empirical studies of[30], [32] support the study.

The results from coefficient of ODA showed that ODA had positive $(0.164745)$ but insignificant (p. $>0.05)$ effect on economic growth. This means that hypothesis two "Official Development Assistance has no significant effect on economic growth of Nigeria" is not rejected. The study therefore concludes that ODA has positive but insignificant effect on economic growth. This is at variance with the study conducted by [8] in Kenya which revealed that ODA had a positive significant relationship with GDP while the study conducted by [27] revealed uncertain effect.

To determine the effect of remittances (REM) on economic growth, the coefficient of REM was used. The result showed the remittances (REM) had a positive $(0.518247)$ and significant (p. <0.05) effect on economic growth in Nigeria. Thus hypothesis three: "Foreign Remittances have no significant effect on economic growth of Nigeria, is rejected. The study therefore concludes that foreign Remittances have significant effect on economic growth of Nigeria. This is supported by the empirical findings in the research conducted in Kenya by [8] and in Nigeria by [27].

The coefficient of EXTDS showed that external debt stock (-0.068816) has a negative but insignificant (p. > 0.05) effect on economic growth in Nigeria implying that hypothesis four: "External debt stock has no significant effect on economic growth of Nigeria" is not rejected. This indicates that external debt stock has no significant effect on economic growth of Nigeria. The studies which posit effect of external debt stock were divergent in their submissions with [9] supporting negative effect while the study by [12] supported positive effect.

In summary, thestudy showed that $71 \%$ of variation in economic growth is accounted for by joint variations in the explanatory variables of international capital inflows. This suggests that capital inflows are valuable tools to enhancing economic growth in Nigeria. Nigeria can further develop its economy if it can encourage the use of international capital. However, the study showed that FDI and REM are international capital inflow variables that have significant and positive effect on economic growth. This indicates that increased use of FDI and Remittances (REM) in Nigeria will lead to higher growth in Nigeria's Gross Domestic Product.Thus, invited external resources coming inform of monetary repatriation, technical inputs and technological transfers have significantly contributed to the growth of Nigerian economy. This result is in line with the theory of this study. It also supported existing empirical studies[28],[32],[33],[36].

\section{Conclusion and recommendations}

International capital inflows have grossly enhanced economic growth of Nigeria. About $71 \%$ of economic growth variations are explained by international capital inflows. This implies that international capital flow is one major contributor to Nigeria's economic growth. However, FDI and Remittances are the main economic growth enhancers in Nigeria, while exchange rate can have a devastating effect on Nigeria's growth.

Since the result suggest that external debt so far has negative effect on growth of the Nigerian economy, the policy makers should forthwith discourage the use of external debt in Nigeria. Since theories have supported that these capital channels should boost growth, it is pertinent that Nigeria government should investigate the spending pattern of the funds obtained through external debts and ODA. More so, the enabling environment has to be made available for FDI to thrive. Government should provide adequate security for lives and property and some needed infrastructure like roads, Electricity and even tax holidays to technologically advanced economies which are mostly the custodians of FDIs. 


\section{References}

[1] M. L. Jhingan,. The Economics of Development and Planning. Delhi: Vrinda Publications Limited 2005.

[2] U. Aurangeb, andA. Haq, Impact of Foreign Capital Inflows on Economic Growth in Pakistan, European Journal of Economics, Finance and Administrative Sciences, Vol. 46, pp. 6 - 12, 2012

[3] M. C. Ekwe, andO. I. Inyiama. Foreign capital flows and growth of the Nigeria economy: An empirical review. International Journal of Economics and Finance, Vol. 6, No. 4, pp. 103 - 109. 2014.

[4] B.C.Ezirim, and M. I.Muoghalu, Output-debt relation in an emerging economy- African experience. Journal of Management Research, Vol. 6, No. 1, pp. 3-17, 2006.

[5] O. RIheke,The effect of remittances on the Nigerian economy. International Journal of Development and Sustainability, Vol. 1 No. 2, pp. 614-621, 2012.

[6] E. Nkoro andA. O. Furo, Foreign capital inflows and economic growth in Nigeria: An empirical approach. Academic Journal of Interdisciplinary Studies, Vol. 2, pp. 55-71, 2012.

[7] E. B.Udah, Remittances, human capital and economic performance in Nigeria. Journal of Sustainable Development in Africa Vol13, No. 4, pp.76-87, 2011.

[8] A. M. Makori, A. Kagiri, and K. Ombui, Effects of external capital inflows on the economic growth in Kenya. Prime Journal of Social Science, Vol. 4, No. 11, pp. 1140-1149, 2015.

[9] E. Nkoro,andA. K.Uko, Foreign capital inflows and economic growth in Nigeria: An empirical approach. Asian Journal of Empirical Research, Vol. 2, No. 5, pp149-161, 2013.

[10] S. Ali, Foreign capital flows and economic growth in Pakistan: An empirical analysis. World Applied Sciences Journal, Vol. 29, No. 2, pp. 193-201, 2014.

[11] N. Sethi and K. U. S. Patnaik,.Impact of international capital flows on India's economic growth. 2005, doi=10.1.1.551.6863\&rep=rep1\&type $=$ pdf.

[12] E. E.,Chigbu, C. P.Ubah, andU. S.Chigbu, Impact of capital inflows on economic growth of developing countries. International Journal of Management Science and Business Administration, Vol. 1, No. 7, pp. 7-21. 2015.

[13] S. O. N.Ibenta, Investment analysis and financial management strategy. Enugu: Institute for Development Studies, 2005

[14] K.L. Gupta, andM. A.Islam, Foreign capital, savings band growth: An international cross section study .D. Reidel Publishing Company, Dordrecht-Holland/Boston, USA, 1983.

[15] K. H.Danja,Foreign direct investment and the Nigerian economy. American Journal of Economics, Vol. 2, No. 3, pp 33-40, 2012.

[16] R. E., Lipsey, The role of foreign direct investment in international capital flows, NBER Working Paper No. 7094.1999.

[17] H. Girma. The impact of foreign aid on economic growth: Empirical evidence from Ethiopia (1974-2011) using ARDL approach Journal of Research in Economics and International Finance, Vol. 4, No. 1, pp. 1 - 12.2015.

[18] E. L.Inanga, and E. Mandah,. Foreign aid finance and economic development: The case of two foreign aid financing agencies in Zambia. International Research Journal of Financial Economics, Vol., 14, pp. 322-358. 2008.

[19] D. Kihangire, andM. Katarikawe. The impact of remittances on macroeconomic stability and financial sector deepening: Opportunities and challenges for Uganda. Working Papers No. 67. 2008

[20] T. Tewolde, Remittances as a tool for development and reconstruction in Eritrea. An economic analysis. Journal of Middle Eastern Geopolitics, Vol. 1, No.2, pp. $14-24.2005$

[21] J. O. Oucho. African diaspora and remittance flows: Leveraging poverty? Centre for Research in Ethnic Relations. University of Warwick, United Kingdom. Paper prepared for the African Migration Yearbook 2008. 
[22] O. E. Obademi, An analysis of the impact of external debt on banks' performance: Evidence from Nigeria. British Journal of Arts and Social Sciences, Vol. 12, No. 1, pp. $37-51.2013$

[23] M. Arnone, L. Bandiera,and A. F. Presbitero, External debt sustainability: Theory and empirical evidence. 2005. Retrieved from http://www3.unicatt.it/unicattolica/Dipartmenti/DISES/allegati/ArnoneBandieraPresbiter033.pdf.

[24] S. I. Ajayi, andM. S. Khan, External debt and capital flight in Sub-Saharan Africa, International Monetary Fund.2000.

[25] R. F. Harrod, An essay in dynamic theory. Economic Journal, Vol49, pp. 14-33.1939.

[26] E. D. Domar, Capital expansion, rate of growth and employment. Econometrica, Vol. 14, pp. 137-150.1946.

[27] I. D. Raheem and O. A.Adeniyi,Capital inflows and outflow and economic growth in Sub-Saharan Africa. International Journal of Economics and Business Research, Vol 10, No. 1, pp. 66 - 80, 2015.

[28] C. A,Adegboye, A. O.Ogbebor, and M. I.Egharvba,External capital flows and economic growth in Nigeria. JORIND, Vol. 12, No. 2, pp. $91-98.2014$.

[29] J, E. C.Nweke. Impact of foreign capital inflows on economic growth and self-employment in Ethiopia. Masters programme in Economic History, School of Economics and Management, Lund University.2015

[30] J. Ferdaous, Impact of remittances and FDI on economic growth: A panel data analysis. Journal of Business Studies Quarterly, Vol. 8, No. 2, Pp. $58-77,2016$.

[31] S. O.Olaleye, Impact of capital flows on economic growth in Nigeria. International Journal of Economics, Commerce and Management, Vol. III, No. 5, 1285 - 1304.2015.

[32] E. I.Okafor,H. C.Ezeaku andG. E.Eje, Foreign investment and its effect on the economic growth in Nigeria: A triangulation analysis. IOSR Journal of Economics and Finance (IOSR-JEF), Vol.6, No. 4, Pp. 01-07.2015.

[33] B.A.Akanyo, andH. A.Ajie,Impact of capital flows on the Nigerian economy in a liberalized environment, 1981-2012.International Journal of Business Finance and Management Research, 3, 6-18. 2015.

[34] S. I. Kanu, Foreign capital inflows and economic growth in Sub-Saharan Africa: A study of selected countries. Research Journal of Finance and Accounting, Vol. 6, No. 1, pp. 52 - 64,2015.

[35] Orji, Uche\&Ilori (). Foreign capital inflows and growth: An empirical analysis of WAMZ experience. International Journal of Economics and Financial Issues, Vol. 4, No, 4, pp. 971-983. 2014.

[36] I. G.Okafor,S. Ugwuegbe, andH. C.Ezeaku,Foreign capital inflows and Nigerian economic growth nexus: A Toda Yamamoto approach. European Journal of Accounting, Auditing and Finance Research, Vol. 4, No. 3, pp. 16-26, 2016. 